\title{
Effects of Molecular Weight on the I sothermal Crystallization of Poly(1-butene)
}

\author{
Stefano Acierno and Nino Grizzuti* \\ Dipartimento di Ingegneria Chimica, Università di Napoli "Federico II", Piazzale Tecchio 80, \\ I-80125 Napoli, I taly
}

\section{H. Henning Winter*}

Department of Chemical Engineering and Polymer Science and Engineering Department, University of Massachusetts, Amherst, Massachusetts 01003

Received J anuary 10, 2002; Revised Manuscript Received March 22, 2002

\begin{abstract}
The effect of molecular weight on the crystallization behavior of isotactic poly(1-butene) (iPB) under quiescent, isothermal conditions is investigated by rheological and optical microscopy techniques. Small-amplitude oscillatory shear experiments are used to determine the critical gel behavior of the crystallizing melt. The resulting critical gel time is related to the crystallization kinetics during the early stages of the process. While molecular weight does not substantially affect the gel characteristic time, the rheological parameters of the crystalline critical gel are found to be strongly dependent upon molecular weight, thus suggesting that the viscoelastic behavior in the early stages of crystallization is dictated by the molecular properties of the amorphous phase. Optical microscopy observations provide quantitative information on the growth rate and the morphology of the crystallites. It is found that the molecular weight has no effect on the iPB growth rate. Conversely, molecular weight affects the morphological transition from spherulites to square-shaped (hedrites) crystallites. The coupled effects of temperature and molecular weight on the spherulite-to-hedrite transition are presented in the form of a phase diagram which quantitatively confirms recent observations made by $\mathrm{Fu}$ et al.
\end{abstract}

\section{Introduction}

Polymer crystallization is a complex phenomenon that crucially affects the final properties of processed polymeric materials. The quality and quantity of crystallization, i.e., the number, size, shape, and crystalline form of the crystallites, depend on a number of different parameters and material properties. Among them, molecular weight (MW) and molecular weight distribution (MWD) are known to play an important role when flowinduced crystallization conditions are considered. ${ }^{1}$ The situation is more controversial, however, when the role of MW and MWD on the quiescent crystallization rate is considered.

Systematic quantitative studies of the effect of MW on quiescent crystallization kinetics are not common in the literature. Furthermore, the attention has been mainly focused either on the growth phase or on the overall crystallization kinetics rather than on the nucleation stage. In crystallization of polyolefins the dependence of the overall crystallization rate and of linear growth rate on the molecular weight is not yet clear. Hoffman ${ }^{2}$ and Hoffman and Miller ${ }^{3}$ observed a growth rate of polyethylene that decreases as $\mathrm{MW}$ increases. Fatou et al. ${ }^{4,5}$ studied the overall crystallization rate of polyethylenes over a wide MW range. Their results showed a peculiar behavior: as MW increases, the half crystallization time, $t_{1 / 2}$, decreases, then passes through a minimum, and increases again to a MWindependent plateau. I sotactic polypropylene does not show any systematic dependence of growth rate and overall crystallization rate on MW. ${ }^{6}$ F or isotactic poly(1-butene) (iPB), which is the material studied in the present work, Cortazar and Guzman ${ }^{7}$ found that the overall crystallization rate increases with increasing molecular mass, whereas Monasse and Haudin ${ }^{8}$ measured a MW-independent linear growth rate.

It is well-known that polymers can give rise to different crystalline forms, depending on the thermal path conditions applied. iPB, for example, may exist at least in three crystal modifications: a rhombohedral one, form I, a tetragonal one, form II, and an orthorhombic one, form III. Cooling from the melt results in the growth of unstable crystals of form II, which transform into form I crystals when keeping the polymer at room temperature for several days.9,10 Form III has been observed in the case of crystallization from solution. ${ }^{11}$

The effect of MW on the crystalline forms and on crystallite morphology is a much less studied subject. Physical intuition would suggest that MW plays no role in determining the crystallite molecular structure. However, recent observation in polarized light microscopy and WAXS on iPB samples showed different crystal morphologies of the form II, namely, a spherulitic morphology, and a hedritic one. ${ }^{12}$ It was found that the transition from spherulites to hedrites depends on both temperature and molecular weight. Such a transition was attributed to the coupling between the temperaturedependent lamellar characteristic length and the MWdependent polymer coil dimension.

In recent years, the use of rheological techniques to monitor polymer crystallization has gained increasing consensus. In particular, the work of Winter's group ${ }^{13,14}$ has shown that the early stages of this liquid-solid transition can be viewed as a physical gelation process. The polymer at its gel point marks the transition between liquid and solid.

Materials at the gel point (critical gels) exhibit a distinctive rheological behavior. The relaxation modulus 
Table 1. Molecular and Rheological Parameters of IPB Samples

\begin{tabular}{lllll}
\hline \multicolumn{1}{c}{ polymer } & iPB398 & iPB295 & iPB177 & iPB116 \\
\hline $\mathrm{M}_{\mathrm{n}}[\mathrm{g} / \mathrm{mol}]$ & 106000 & 64500 & 54000 & 37200 \\
$\mathrm{M}_{\mathrm{w}}[\mathrm{g} / \mathrm{mol}]$ & 398000 & 295000 & 177000 & 116000 \\
$\mathrm{M}_{\mathrm{w}} / \mathrm{M}_{\mathrm{n}}$ & 3.8 & 4.6 & 3.3 & 3.1 \\
$\% \mathrm{mmmm}$ & 82.7 & 81.7 & 79.5 & 79.5 \\
$\mathrm{MFI}[\mathrm{dg} / \mathrm{min}]$ & 1.8 & 2.5 & 18.5 & 200 \\
$\mathrm{~T}_{\mathrm{m}}\left[{ }^{\circ} \mathrm{C}\right]$ & 138.4 & 134.8 & 134.0 & 130.4 \\
$\eta_{0}\left(140^{\circ} \mathrm{C}\right)[\mathrm{Pa} \cdot \mathrm{s}]$ & 53400 & 17900 & 3650 & 923
\end{tabular}

$\mathrm{G}(\mathrm{t})$ and the relaxation spectrum $\mathrm{H}(\lambda)$ reduce to power law form: ${ }^{15}$

$$
\begin{gathered}
\mathrm{G}(\mathrm{t})=\mathrm{St}^{-\mathrm{n}} ; \text { for } \lambda_{0}<\mathrm{t}<\infty \\
\mathrm{H}(\lambda)=\frac{\mathrm{S}}{\Gamma(\mathrm{n})} \lambda^{-\mathrm{n}} ; \text { for } \lambda_{0}<\mathrm{t}<\infty
\end{gathered}
$$

where $\mathrm{S}$ is the gel stiffness, $\mathrm{n}$ is the relaxation exponent, $\lambda$ is the relaxation time, $\lambda_{0}$ delimits the crossover to some faster dynamics, and $\Gamma(\mathrm{n})$ is the gamma function. The gel equations (1) or (2) allow for prediction of all linear viscoelastic properties of the critical gel. In particular, as a consequence of the power law dynamics, the loss tangent in the low-frequency region is predicted to be frequency independent at the gel point:

$$
\tan \delta=\frac{\mathrm{G}^{\prime \prime}}{\mathrm{G}^{\prime}}=\tan \left(\frac{\mathrm{n} \pi}{2}\right) \neq \mathrm{f}(\omega) ; \text { for } 0<\omega<1 / \lambda_{0}
$$

where the phase shift angle, $\delta$, is directly related to the slope of the dynamic moduli. Equation 3 provides the most reliable method to rheol ogi cally determi ne the gel time and the parameters $\mathrm{S}$ and $\mathrm{n}$ of the critical gel.

Measurements of the viscoel astic moduli during crystallization can be used to detect the gel point, which in turn can be related to the crystallization kinetics during the initial crystallization stages. A major open question, however, is the relation between the critical gel behavior and the underlying microstructure in a crystallizing polymer. Horst and Winter, ${ }^{16}$ for example, proposed three different connectivity structures at the gel point. At present, however, neither the local structure nor the meaning of the gel parameters $\mathrm{S}$ and $\mathrm{n}$ of a crystalline gel is understood in great detail.

The aim of this work is to clarify the effects of molecular weight on the crystallization behavior of isotactic poly(1-butene) over all stages of the crystallization process. To this end, different experimental techniques have been employed. On one hand, the critical gel concept has been exploited to rheologically characterize the early stages of crystallization. On the other hand, optical microscopy has been used to determine the subsequent growth rate, at least up to the point where crystallite impingement limits the direct optical method. Optical mi croscopy has also allowed to quantitatively characterize a temperature-dependent morphology transition from spherulites to hedrites and its dependence upon polymer molecular weight.

\section{Experimental Section}

Materials. Four commercial grades of isotactic poly(1butene) (iPB) from Shell served as model polymers for the study. A summary of the sample physical properties is given in Table 1. The iPBs do not contain any significant amount of nucleating agents. The weight-average molecular weight ranged from about 100000 to about $400000 \mathrm{Da}$. The polydispersity index $M_{w} / M_{n}$ was similar for all samples, falling in the range 3.1-4.6. The index of tacticity was al so essentially the same for all molecular weights investigated.

Disk-shaped samples for all experiments were prepared from the as-received pellets by molding at $160{ }^{\circ} \mathrm{C}$ for $5 \mathrm{~min}$ under vacuum in a Carver laboratory press. F or the rheometer and the optical cell, samples were always preheated at 160 ${ }^{\circ} \mathrm{C}$ for 5 min to guarantee a complete melting of the crystalline phase. Both rheological and optical measurements were carried out under a dry nitrogen atmosphere to minimize polymer degradation.

Methods. Rheological tests were performed in torque controlled rotational rheometers, Stresstech of ATS Rheosystem and SR200 of Rheometric Scientific. In both instruments, a parallel plate configuration was used (plate diameter $25 \mathrm{~mm}$, gap thickness $1 \mathrm{~mm}$ ).

For the optical microscopy observations, the sample was placed in a Linkam optical cell (CSS 450, Linkam Scientific Instruments Ltd.) and observed by a polarizing microscope (Universal polarizing microscope ZPU 01, Carl Zeiss Inc.). The microscope was equipped with a CCD camera and different magnification objectives. Microscope images were directly digitized by means of a frame grabber (DT-3152, Data TransIation Inc). Quantitative analysis of the digital images was carried out by a suitable software (Scion I mage, Scion Corp.). In this case, a sample thickness of $0.2 \mathrm{~mm}$ was chosen as a good compromise between the opposite needs of clear optical observations of the growing spherulites and of experimental conditions close to those used for the rheological measure ments.

To determine the gel time under isothermal, quiescent conditions, the following experimental protocol was used. After preheating of the sample at $160^{\circ} \mathrm{C}$, a rapid cooling $(20 \mathrm{~K} / \mathrm{min})$ down to the test temperature was applied. The polymer was then subjected to a continuous sequence of frequency sweeps. The gel time was determined as the time where $\tan \delta$ is frequency-independent, at least in the low frequency region. At the test temperatures used in this work, it was always verified that the cooling time was much shorter than the characteristic nucleation time, thus ensuring that the crystallization took place under practically isothermal conditions. It was also verified that the mutation time, that is, the ratio between the time necessary for a single frequency sweep and the crystallization characteristic time, was al ways sufficiently small, so that the gel time could be determined with good accuracy. ${ }^{17}$

Since the crystallization rate of iPB is extremely sensitive to the crystallization temperature, an absolute and relative temperature calibration of the different apparatuses was performed. In particular, the thermocouples of the optical cell and of the ATS rheometer were calibrated using high-purity standard materials that are characterized by sharp, wellknown melting temperatures $\mathrm{T}_{\mathrm{m}}$. Naphthalene $\left(\mathrm{T}_{\mathrm{m}}=80.3^{\circ} \mathrm{C}\right)$, benzoic acid $\left(T_{m}=122.4^{\circ} \mathrm{C}\right)$, and indium $\left(T_{m}=156.6^{\circ} \mathrm{C}\right)$ were used as calibration standards. ${ }^{18} \mathrm{~A}$ thin layer of solid material was compressed between the parallel plates of the two apparatuses, and a linear temperature ramp was applied. The melting point was determined as the temperature at which the applied compression force dropped to zero, in the rheometer, or the sample edge started to move under the action of the compression force in the optical cell. To remove the effect of heating rate on the melting temperature, three different rates ware used $(0.5,1$, and $2 \mathrm{~K} / \mathrm{min})$, and the results were extrapolated to zero heating rate.

\section{Results and Discussion}

Rheology. For temperatures ranging from 120 to 220 ${ }^{\circ} \mathrm{C}$, the iPB samples show the typical linear viscoelastic behavior of a modestly entangled polymer melt (see Figure 1). Time-temperature superposition collapses the data into a single master curve for each MW. In Figure 1, the reference temperature of $140^{\circ} \mathrm{C}$ has been used. The activation energy for the temperature shift is $E / R=6.3 \times 10^{3} \mathrm{~K}$. The zero-shear-rate viscosity is a 


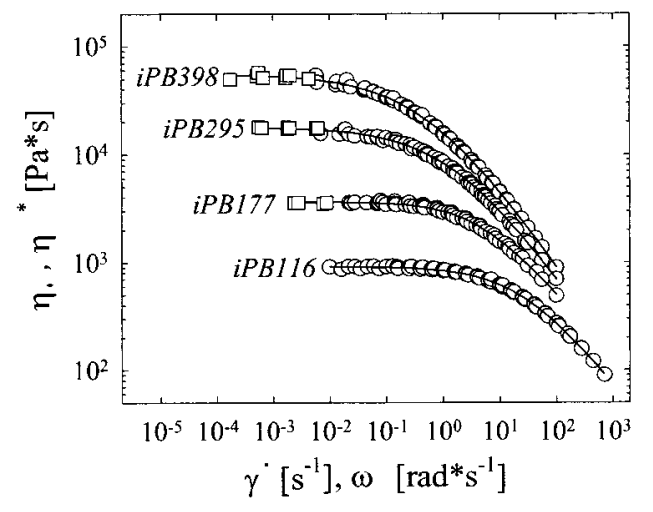

Figure 1. Viscosity master curves of the four isotactic poly(1-butene) samples at the reference temperature of $140^{\circ} \mathrm{C}:(\mathrm{O})$ $\eta^{*},(\square) \eta$.

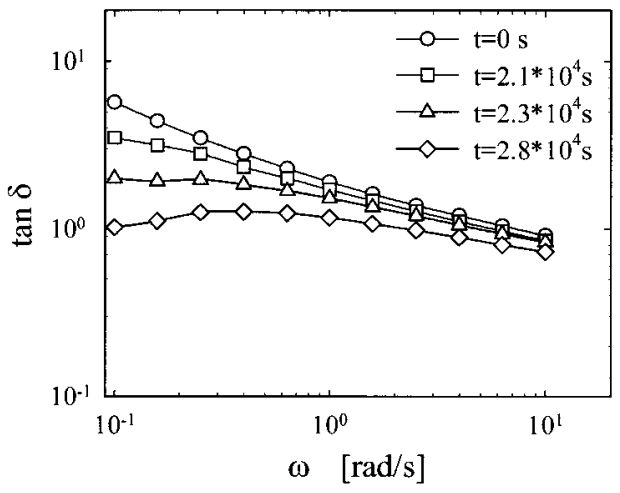

Figure 2. Evolution of the loss angle tangent vs frequency during the isothermal crystallization of iPB177 at $103^{\circ} \mathrm{C}$ under quiescent conditions. The flat region at low frequencies is characteristic of the system at its gel point (critical gel).

strong function of molecular weight, and the crossover to the shear thinning behavior occurs at lower and lower shear rates as the molecular weight increases.

The transition of a crystallizing polymer from the liquid to solidlike state can be viewed as a gelation process. ${ }^{13,14}$ As mentioned in the Introduction, the critical gel state is reached when the viscoelastic phase angle $\delta$ becomes a constant, at least in the low-frequency window. iPB affords the typical tan $\delta$ pattern, as demonstrated in Figure 2 for the iPB177 sample at 103 ${ }^{\circ} \mathrm{C}$. A negatively sloped $\tan \delta$ at short times is characteristic of the polymer in the molten state. An intermediate curve, measured after crystallizati on for about 2.3 $\times 10^{4} \mathrm{~s}$, is clearly frequency-independent in the lowfrequency region, thus corresponding to the gel point. As crystallization proceeds further, the polymer behaves like a solid and, correspondingly, $\tan \delta$ exhibits the typically positive slope at low frequencies.

Measured gel times at the test temperature of 103 ${ }^{\circ} \mathrm{C}$ are summarized in Figure 3 , where $\mathrm{t}_{\text {gel }}$ is plotted as a function of molecular weight. The dotted lines represent the $99 \%$ confidence interval as cal culated over all the experimental data points and for all molecular weights. The band of variation shows that the gel time varies by a factor of less than 2 over all samples, a value which is of the same order of magnitude of the experimental error bars for each MW, al so reported in Figure 3. It can be concluded that, within experimental error, the crystallization rate in the early stages of the process is essentially molecular weight independent.

From the data at the gel point, the rheological parameters of the critical gels were determined, i.e., the

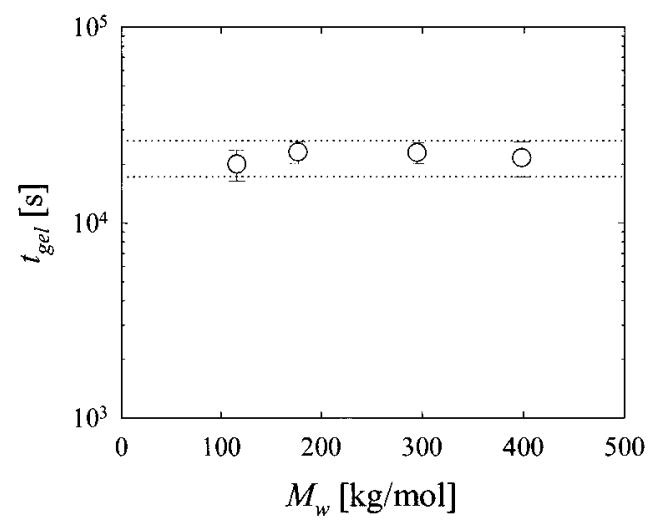

Figure 3. Gel time vs molecular weight for isothermal crystallization at $103{ }^{\circ} \mathrm{C}$ under quiescent conditions. Error bars are standard errors. The dotted lines represent the $99 \%$ confidence interval for the whole set of experimental data (see text).

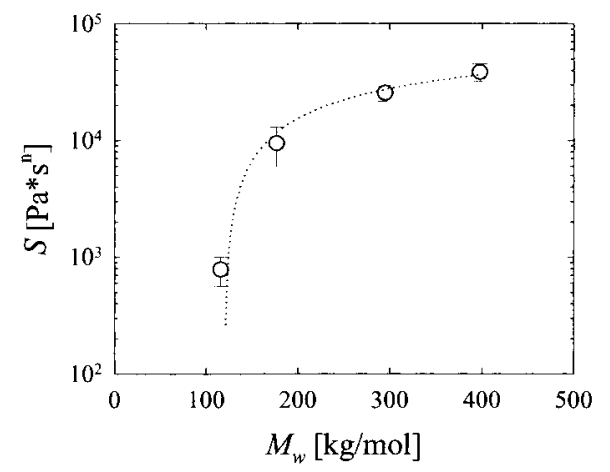

Figure 4. Gel stiffness vs molecular weight at the experimental temperature of $103{ }^{\circ} \mathrm{C}$ (error bars are standard errors).

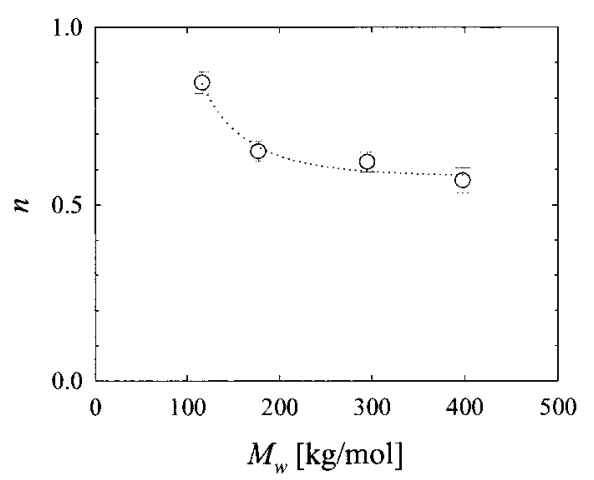

Figure 5. Relaxation exponent vs molecular weight at the experimental temperature of $103^{\circ} \mathrm{C}$ (error bars are standard errors).

gel stiffness $\mathrm{S}$ and the relaxation exponent $\mathrm{n}$ (see eqs 1-3). Their dependence upon molecular weight is shown in Figures 4 and 5. The values of $S$ and $n$ were determined in the low-frequency region, where $\tan \delta$ is frequency-independent. It can be noticed that S strongly increases as molecular weight is increased, whereas $n$ shows the opposite trend. Critical gels get stiffer and relax more slowly when crystallized from higher molecular weight polymers.

The simultaneous increase of the gel strength and decrease of the gel relaxation exponent is a common feature of many systems showing critical gel behavior. ${ }^{17}$ In the case of crystallizing polymers such a trend usually corresponds to a change in the crystallization temperature. Winter and co-workers (see, for example, Pogodina and Winter ${ }^{14}$ ) al ways found that an increase 
of the degree of undercooling would produce stiffer gels, i.e., gels of increasing $\mathrm{S}$ and decreasing $\mathrm{n}$. The data in Figures 4 and 5 correspond to a fixed temperature and to a varying MW. In this case, however, the change in critical gel stiffness cannot only be due to a change in the degree of undercooling. Indeed, moving from the iPB116 to the iPB177 the change in the equilibrium melting temperature (see Table 1 ) is only $3.6 \mathrm{~K}$ while correspondingly a 12 -fold increase in the gel stiffness is observed. Furthermore, the melting temperatures of the two intermediate samples, namely, iPB177 and iPB295, are practically equal ( 134 and $134.8^{\circ} \mathrm{C}$, respectively). Conversely, their stiffness is substantially different.

To explain the experimental results, we speculate that the critical gel properties of the crystallizing samples (at our experimental conditions) do not depend on the crystalline forming phase but mainly on the microstructure of the amorphous phase. One can imagi ne that the critical gel is made of polymer chains partially embedded in the crystalline entities but still strongly entangled with other chains in the melt phase. An analogy could be made with a system of multiarm polymer stars growing from a matrix of linear chains. The formation of even few stars would generate a dramatic increase in the relaxation time of the system, thus producing the gellike behavior. This hypothesis would be consistent with the observation that the time to reach the gel point is essentially molecular weight independent, whereas the critical gel properties do depend on MW. In fact, upon increasing the mol ecular weight, the number and size of crystalline entities necessary to produce the critical gel would be reached in the same time. However, an amorphous phase constituted of longer chains would generate stars with longer arms, thus producing a stiffer critical gel. The proposed picture would al so be consistent with the observed increased gel stiffness upon increasing the degree of undercooling. I $n$ fact, in terms of polymer relaxation times, a decrease in temperature is perfectly equivalent to an increase of the polymer molecular weight. The only difference, in this case, is that temperature affects also (and dramatically!) the rates of nucleation and of crystallization, thus moving the gel point to shorter times.

Growth Rate. The spherulitic growth was observed by optical microscopy in the Linkam cell. To guarantee consistency with the rheol ogical experiments, the same thermal protocol, specified in the previous section, was applied. As expected, during the isothermal crystallization the spherulite radius increases linearly with the crystallization time until impingement. One example is given in Figure 6, where the time evolution of the radius for the iPB398 sample at the temperature of $94.7^{\circ} \mathrm{C}$ is shown. The spherulitic growth rates were calculated from the slope of the spherulite radius vs time.

Growth rates were measured at temperatures between 86 and $105{ }^{\circ} \mathrm{C}$ and are plotted as a function of the crystallization temperature in Figure 7. Despite some scattering of the data, Figure 7 clearly shows that the growth rate is practically independent of the molecular weight. This result is further clarified by Figure 8 , where the growth rate at two crystallization temperatures, namely, 100 and $95^{\circ} \mathrm{C}$, is plotted as a function of molecular weight. Like the gel time results reported in Figure 3, an uncertainty factor of less than 2 can be appreciated at both temperatures. It should be mentioned that the same statistical oscillation is observed

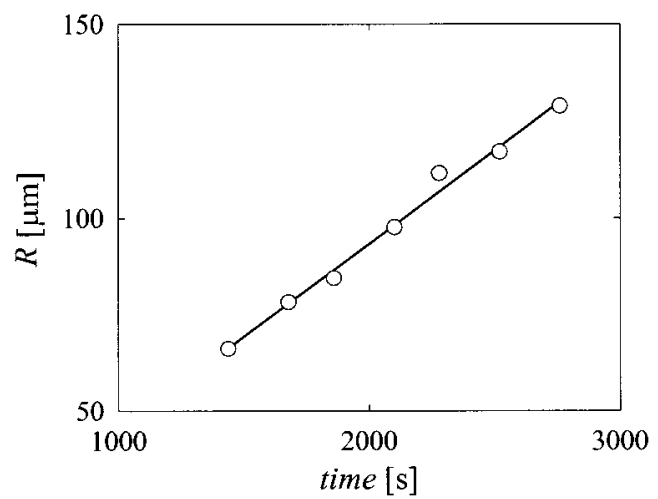

Figure 6. iPB398 at $94.7^{\circ} \mathrm{C}$ : spherulitic radius vs crystallization time.

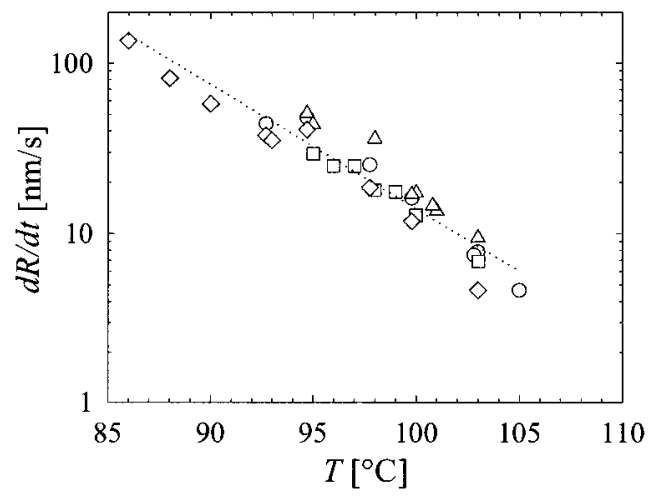

Figure 7. Crystallite growth rate vs crystallization temperature: (O) iPB398; ( $\square)$ iPB295; $(\triangle)$ iPB177; $(\diamond)$ iPB116.

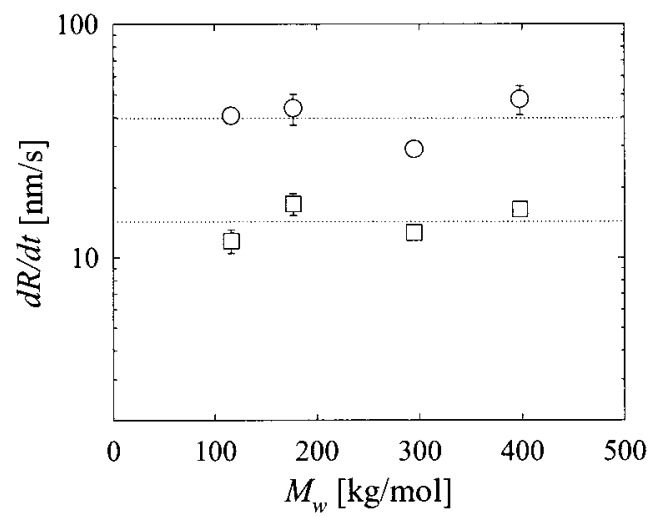

Figure 8. Crystallite growth rates of iPB samples at $(O) 95$ and $(\square) 100{ }^{\circ} \mathrm{C}$. Lines are the mean values at the two temperatures.

when the overall crystallization rate is measured by differential scanning cal orimetry. ${ }^{19}$ The results of Figure 7 are in line with previous measurements by Monasse and Haudin, ${ }^{8}$ leading to the conclusion that also the growth rate of iPB is essentially MW-independent.

Morphology. The combined influence of molecular weight and temperature on the iPB crystal morphology was further investigated by optical microscopy. Figure 9 shows images of the isothermally growing crystallites of iPB295 at different temperatures. The expected spherical crystallites are found at $95{ }^{\circ} \mathrm{C}$ (Figure 9a). At $98{ }^{\circ} \mathrm{C}$ (Figure 9b), however, two coexisting structures can be clearly observed, as square-shaped crystallites grow along with the spherical ones. Such a different crystalline form has been reported very recently by Strobl and co-workers ${ }^{12}$ for iPB samples similar to those used in the present work. The latter authors called them 

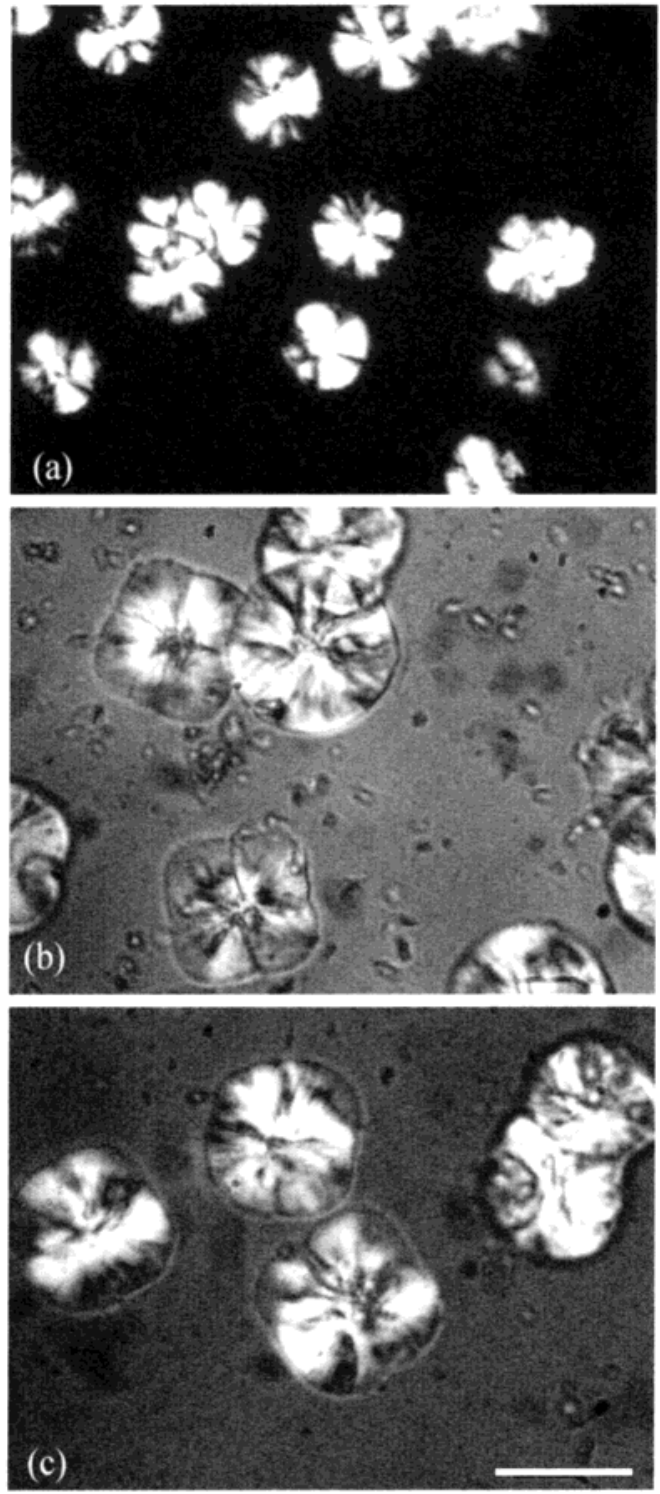

Figure 9. iPB295 crystallizing at (a) 95, (b) 98, and (c) 100 ${ }^{\circ} \mathrm{C}$. The scale bar is $100 \mu \mathrm{m}$.

"hedrites" as they recall single crystals obtained from solution. At even higher temperatures (see Figure 9c corresponding to $100^{\circ} \mathrm{C}$ ), only the hedrites survive. It can be concluded that a critical temperature exists, above which the observed change in crystallite morphology takes place. It should be noted that such a change has no apparent consequences on the crystal growth rate, as confirmed by inspection of Figure 7.

The temperature-dependent formation of the hedrites is strongly coupled to the polymer molecular weight, as confirmed by Figure 10 which refers to the iPB398 sample. In this case, the hedrite morphology is observed above $103{ }^{\circ} \mathrm{C}$. More generally, the critical temperature is found to be an increasing function of the polymer molecular weight. By determining the temperature where the two crystalline morphol ogies coexist for each sample, a phase diagram for the iPB morphology can be constructed. The diagram is shown in Figure 11, where the critical temperature is plotted as a function of molecular weight. The upper left part of the diagram corresponds to the formation of hedrites, whereas in the lower right region the classical spherulites are found.

The phase diagram of Figure 11 represents a quantitative confirmation and an extension of the observa-
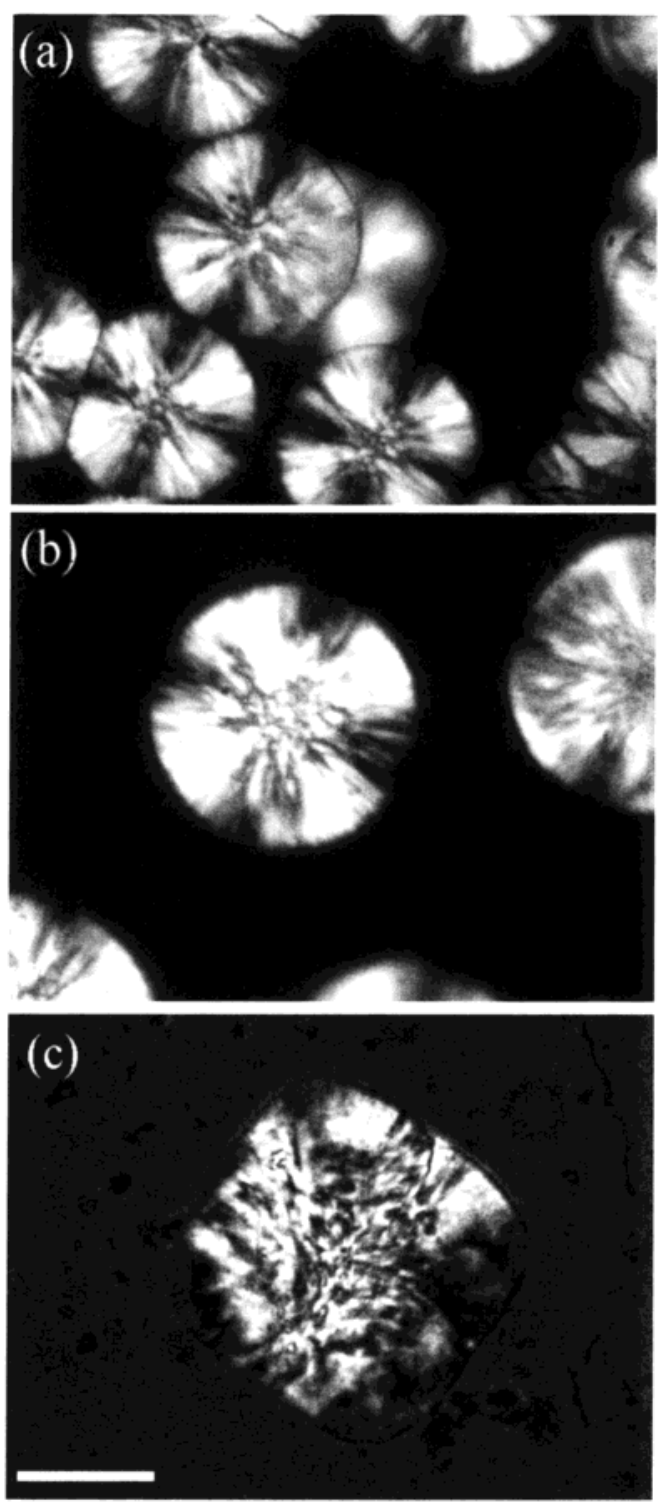

Figure 10. iPB398 crystallizing at (a) 95, (b) 98, and (c) 105 ${ }^{\circ} \mathrm{C}$. The scale bar is $100 \mu \mathrm{m}$.

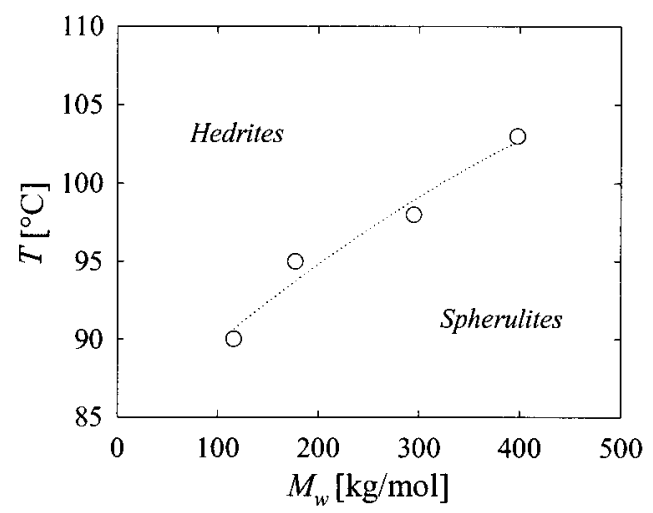

Figure 11. Molecular weight dependence of critical temperature at which the two crystalline morphologies coexist.

tions made by $\mathrm{Fu}$ et al., ${ }^{12}$ who found a difference in critical temperatures for two iPB samples of different MW. To explain this result, $\mathrm{Fu}$ et al. ${ }^{12}$ suggested that the transition between the two crystal line morphologies takes place when the crystalline lamellar size, which increases with increasing temperature, exceeds the MWdependent radius of gyration of the single polymer coil. 
According to the authors, this might correspond to a transition from a chain-folded to a chain-extended crystallization mechanism. Although it cannot be confirmed, such a speculative hypothesis is not contradicted by the phase diagram of Figure 11.

\section{Conclusions}

Critical gel rheological experiments and crystal growth data both indicate that the crystallization rate of the isotactic poly(1-butene) is essentially not affected by the polymer molecular weight. The critical gel result is particularly relevant, as it corresponds to the early crystallization stages, whereas the available literature for iPB refers to either the growth or the overall crystallization rate. The $\mathrm{MW}$ independence of the crystallization rate is confirmed even when a MWdependent crystallite morphology change takes place, from spherulites to hedrites (see below). A MW-invariant crystallization rate under quiescent conditions makes this set of iPB samples an ideal model system to study the effects of molecular weight on flow-induced crystallization. This will be the subject of a forthcoming paper.

Although the gel time is found to be MW-independent, the rheol ogical properties of the critical gel do strongly depend on molecular weight. In particular, the increase in gel stiffness upon increasing molecular weight, coupled to a MW-independent crystallization rate, suggests that the rheology of the critical gel is controlled by the relaxation properties of the amorphous phase. We speculate the similarity between a crystalline critical gel and a polymer melt made of multiarm polymer stars growing from a matrix of linear chains. This picture is also consistent with the well-known increase in critical gel stiffness of crystallizing polymers upon increasing the degree of undercooling.

The optical microscopy results confirm the presence of a morphological phase change in the isothermal crystallization of iPB, recently found by Fu et al. ${ }^{12}$ The transition from a spherulitic to a disklike, hedritic morphology is determined by the coupling between crystallization temperature and molecular weight. In particular, the transition is shifted to higher temperatures upon increasing the polymer MW. As mentioned above, the morphological phase change does not seem to affect the iPB crystallization rate, which remains
MW-independent both below and above the transition temperature.

Acknowledgment. We thank Prof. G. C. Alfonso for providing the samples and making available some unpublished data and Dr. R. Horst for hel pful advice with the rheo-optical experiments at UMass. The work is supported by the I talian Ministry of U niversity (PRIN 1999-2001, "Flow induced crystallization of polymers. Impact on processing and manufacturing properties") and by ExxonM obil Chemicals.

\section{References and Notes}

(1) Duplay, C.; Monasse, B.; Haudin, J .-M.; Costa, J .-L. J . Mater. Sci. 2000, 35, 6093-6103.

(2) Hoffman, J . D. Polymer 1982, 235, 656-670.

(3) Hoffman, J . D.; Miller, R. L. Macromol ecules 1988, 21, 3038 3051.

(4) Fatou, J. G.; Marco, C.; Mandelkern, L. Polymer 1990, 31, 890-898.

(5) Fatou, J. G.; Marco, C.; Mandelkern, L. Polymer 1990, 31, 1685-1693.

(6) Bicerano, J. J . Macromol. Sci., Rev. Macromol. Chem. Phys. 1998, C38, 391-479.

(7) Cortazar, M.; Guzman, G. M. Makromol. Chem. 1982, 183, $721-729$.

(8) Monasse, B.; Haudin, J. M. Makromol. Chem., Macromol. Symp. 1988, 20/21, 295-302.

(9) Natta, G.; Corradini, P.; Bassi, I. W. Makromol . Chem. 1956, 21, 240-244.

(10) Danusso, F.; Giannotti, G. Makromol. Chem. 1965, 88, 149158.

(11) Danusso, F.; Giannotti, G.; Polizzotti, G. Makromol. Chem. 1964, 80, 13-21.

(12) Fu, Q.; Heck, B.; Strobl, G.; Thomann, Y. Macromolecules 2001, 34, 2502-2511.

(13) Lin, Y. G.; Mallin, D. T.; Chien, J. C. W.; Winter, H. H. Macromolecules 1991, 24, 850-854.

(14) Pogodina, N. V.; Winter, H. H. Macromolecules 1998, 31, 8164-8172.

(15) Winter, H. H. In Encyclopedia of Polymer Science and Engineering, 2nd ed.; Mark, H. F., Bikales, N. M., Overberger, C. C., Menges, G., Kroschwitz, J. I., Eds.; Wiley-Interscience: New York, 1989; Suppl. Vol., p 343.

(16) Horst, R. H.; Winter, H. H. Macromol ecules 2000, 33, 75387543.

(17) Winter, H. H.; Mours, M. Adv. Polym. Sci. 1997, 134, 165234.

(18) Lage's Handbook of Chemistry; Dean, J . A., Ed.; McGrawHill: New York, 1999.

(19) Alfonso, G. C. Personal communication, 2001.

MA0200423 